\title{
ENHANCED QUALITY OF SERVICE STRATEGY FOR IMPROVING NETWORK COVERAGE IN IOT APPLICATIONS
}

\author{
Dr. D. I. George Amalarethinam ${ }^{1}$, Ms. P. Mercy $^{2}$ \\ Bursar \& Director (MCA), Associate Professor, ${ }^{2}$ Research Scholar \\ PG \& Research Department of Computer Science \\ Jamal Mohamed College (Autonomous), Affiliated to Bharathidasan University, \\ Tiruchirappalli - 620020, Tamil Nadu, India. \\ E-mail: 1di_george@ymail.com, ${ }^{2}$ mercyvijay23@gmail.com
}

DOI: $10.51201 / J U S S T / 21 / 05201$

http://doi.org/10.51201/JUSST/21/05201

\begin{abstract}
The Internet of Things (IoT) is a network that includes physical things capable of aggregating and communicating the electronic information. With the advancement in Wireless sensor network, IoT provides highly efficient communication for various real-time applications. IoT networks are a large-scale network where routing can be improved by focusing on Quality of Service (QoS) Parameter. Network coverage can be enhanced by hierarchical clustering of the nodes which increases the network lifetime. The proposed algorithm Enhanced Fuzzy Based Clustering and Routing Algorithm (EFCRA) performs distance and energy-based cluster head selection to find a new path from source to destination. The algorithm uses Fuzzy c-means clustering to provide optimization in forming cluster centres. The cluster head $(\mathrm{CH})$ is identified based on the minimum distance and maximum energy of the sensor node. The cluster head is updated when its energy is lesser than the threshold value. The distance between sensor nodes and its $\mathrm{CH}$ node and then to the destination is computed using Dijkstra's algorithm. The proposed routing strategy provides improved network coverage and throughput which extends the lifetime of the IoT network.
\end{abstract}

Keywords: Internet of Things, Quality of Service, Hierarchical Clustering, Routing, IoT Coverage.

\section{Introduction}

Internet of Things (IoT) is defined as inter-connection of things that has physical and virtual attributes and communicate with each other to exchange data using internet. Wireless sensor network (WSN), cloud computing, big data, embedded systems, communication protocols are considered to be enabling technologies of IoT. IoT enables these intelligent objects to collect and exchange data [1]. It is a huge dynamic network based on the principle that the things or device can interact with each other to accumulate and distribute data about their context. In IoT network, sensors are used to sense the value from the context in which it 
is deployed. The sensed value or the data is transmitted to the base station [2]. During transmission of data, energy of the sensor node gets depleted which results in the loss of communication and desired quality of service cannot be obtained. The Quality of Service (QoS) can be obtained when the network traffic flow is maintained without any congestions. The enhancement in the QoS parameters lead to the betterment of network performance [3]. Fig. 1 shows the basic architecture of IOT based WSN.

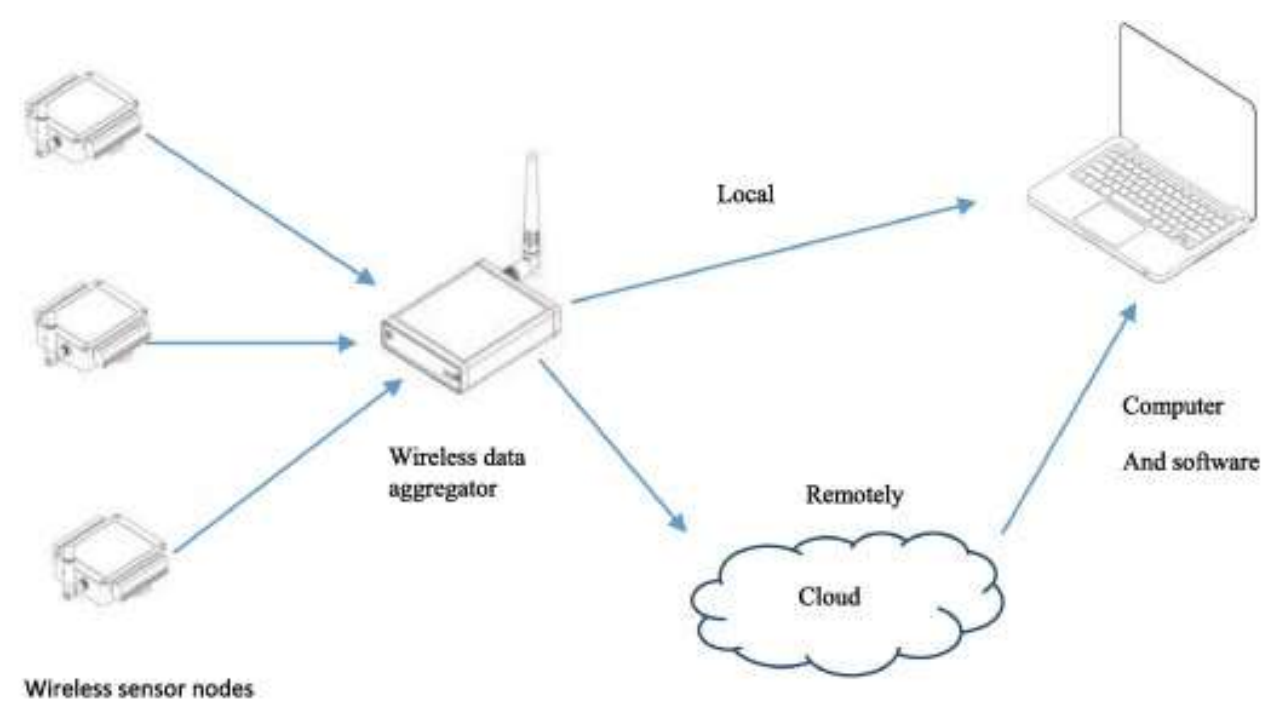

Fig.1 IoT Based Wireless Sensor Network

Many real-time IoT applications, such as smart cities, weather forecasting, smart irrigation and coal mine safety monitoring system etc. need QoS based routing strategy to reduce the data loss and to increase the data transmission rate. QoS based IoT network helps to achieve more deterministic network behaviour, so that the information carried by the network can be better delivered and network resources can be better utilized [4]. QoS highly depends on the most common parameters like Channel bandwidth, Packet delay, network life time, jitter, packet losses, reliability, throughput, energy and coverage [5]. Clustering of nodes in an IoT network can enhance the IoT coverage. Clustering of nodes reduces the energy consumed during the communication of data to the base station. Due to the minimum energy consumption, the lifetime of the IoT networks can be extended [6].

IoT is a self-organized dynamic network [7]. In WSNs for IoT, the overall network performance can be improved by minimizing the energy consumption [8]. Clustering of nodes in the network helps to stabilize the network topology which will improve the scalability and coverage of the networks. The appropriate Cluster head $(\mathrm{CH})$ selection among the clusters results in improved network lifetime by maintaining the average remaining 
energy of the $\mathrm{CH}$ nodes and member nodes (MN) [9]. Most IoT applications enhance its routing decision based on the QoS parameters like minimum end-to-end delay, maximum throughput and minimum energy consumption [10].

The proposed strategy uses Fuzzy C means clustering algorithm (figure 4) to find centroids in the hierarchical structure of node deployment and Greedy Heuristic optimization technique to identify the cluster head. The strategy computes the minimum distances between cluster head and base station. It also checks the energy of the cluster head while transmitting data to the base station. Thus a new algorithm is proposed to improve the performance of the network based on the $\mathrm{CH}$ selection. Conventional works use distance, priority and probability based cluster head selection process to perform the communication between source and destination. This may reduce the performance of the system caused by power unavailability due to high data transmission of particular nodes. To avoid this, energy and distance based cluster head selection algorithm is proposed to determine a best path from source to destination.

The work is organized as follows: Section II is dealt with related works of clustering technique. Fuzzy $\mathrm{C}$ mean clustering of nodes and greedy heuristic of $\mathrm{CH}$ selection is explained in section III. Simulation results are discussed in section IV. Finally conclusion can be observed from section $\mathrm{V}$.

\section{Related Works}

In IoT network, routing is based on three major components such as Network structure, Route Discovery and Protocol operation. Network structure is the arrangement or deployment of nodes in the IoT network. It is classified into hierarchical or vertical based routing, flat or horizontal based routing and location-based routing. Many large scale IoT application is based on hierarchical based routing strategy. Clustering and cluster head selection improves network scalability, life time and reduce the energy consumption of the networks.

Lina et al., [11] has discussed various types of clustering techniques of wireless sensor network that enhances the QoS and Quality of experience (QOE) in IoT. Clustering algorithm can be classified in two phases such as $\mathrm{CH}$ election and $\mathrm{CH}$ formation or creation. Routing between member nodes and $\mathrm{CH}$ is called intra-cluster routing. Routing between 
inter- $\mathrm{CH}$ is inter-cluster routing. The routing scheme can be classified as single hop with clustering and multi hop with clustering.

Yoongsung et al., [12] has proposed a mechanism that involves multihop clustering of nodes for IoT network to improve the coverage area and network life time. It minimizes the number of required transmission from the node to the internet server by reducing the number of coordinator nodes. The coordinator nodes called $\mathrm{CH}$ is obtained using greedy heuristic based on maximum hop count constraint. Energy of the IoT node is not taken into consideration.

Amol V.Dhumane et al., [13] has suggested an optimal routing algorithm. The algorithm uses k-means clustering algorithm in which the membership of nodes belong to one cluster. Genetic algorithm is used to find the optimal path by finding the energy cost of the $\mathrm{CH}$ node and trust level of the sensor nodes.

Lina xu et al.,[14] has proposed a a clustering algorithm that can select $\mathrm{CH}$ based on energy consumption and deployment of sensors. It enhances the network lifetime and its coverage. Angha Rajput et al., [15] has suggested a WSN based IoT applications for agricultural domain. It uses FCM algorithm for clustering of homogeneous sensor nodes and selects $\mathrm{CH}$ based on perceived probability to improve network scalability. Kavita Jaiswal and Veena Anand., [16] has suggested multi-path routing protocol based QoS parameter like throughput, end to end delay and packet delivery ratio. The optimal cost factors namely lifetime and congestion of a node is used to determine path between source and destination. The less congested path results in minimum delay and the protocol delivers the packet to the destination thus attains the minimum latency.

\section{Proposed Work}

The proposed algorithm Fig.4 enhances the Quality of service in IoT network by improving its coverage, throughput and network life time. Clustering plays a vital role in improving the network coverage and lifetime of a large scale network. When each node in the network sends data packet directly to the base station will result in the maximum energy consumption that lead to death of the nodes. The proposed EFCRA algorithm finds the cluster centre using Fuzzy C mean clustering method. The algorithm enhances the Quality of service in IoT application by clustering the nodes based on distance and energy. The $\mathrm{CH}$ node can be updated when the energy of $\mathrm{CH}$ node is minimum than the threshold value. It also computes the best path between destination and the cluster head which results in the excellent 
coverage and throughput for improving IoT network lifetime. The proposed EFCRA algorithm flow diagram is shown in figure 3.

\section{A. Finding Cluster Centres}

Most real time applications of IoT follows dynamic and reactive route discovery that a node can belong to more than one cluster in the network. Fuzzy c-means (FCM) is a soft and unsupervised clustering method. In k-means clustering algorithm a node can belong to only one cluster. FCM is better than k-means algorithm. Conventionally, the number of cluster is given as an input to FCM algorithm by the user. The proposed work determines the cluster size based on the network size and the number of sensor in each grid. Initially network area of size $\mathrm{N}$ is divided equally into grids. The number of grid in the network is considered as Grid Count (GC). Grid Density is computed by using the equation

$$
G D=\frac{N C\left(g_{i}\right)}{G C} * \sqrt{N}
$$

where GD is the Grid Density, NC represents the Node Count, GC represents the Grid Coun, $\mathrm{N}$ denotes the network size, $\mathrm{g}_{\mathrm{i}}$ denotes $\mathrm{i}^{\text {th }}$ grid in the network, $\mathrm{NC}\left(\mathrm{g}_{\mathrm{i}}\right)$ represents node count in the $\mathrm{i}^{\text {th }}$ grid. The steps for determining the cluster size is given in [17]. Fig.3 shows the example of Fuzzy C-Means clustering of nodes.

The FCM algorithm is based on minimizing the objective function of $\mathrm{J}_{\mathrm{m}}$, and this function depends on the cluster centres and the fuzzy properties of the cluster inputs. The objective function is defined as follows [19]:

$$
\operatorname{Min} J_{m}=\sum_{j=1}^{N} \sum_{i=1}^{C} \mu_{i j}^{m} d^{2}
$$

where $\mathrm{C}$ denotes number of clusters,

$\mathrm{d}$ is the Euclidean distance between $\mathrm{j}^{\text {th }}$ node and $\mathrm{i}^{\text {th }}$ cluster center $\mathrm{m}$ (constant value) is the degree of fuzzifier. 


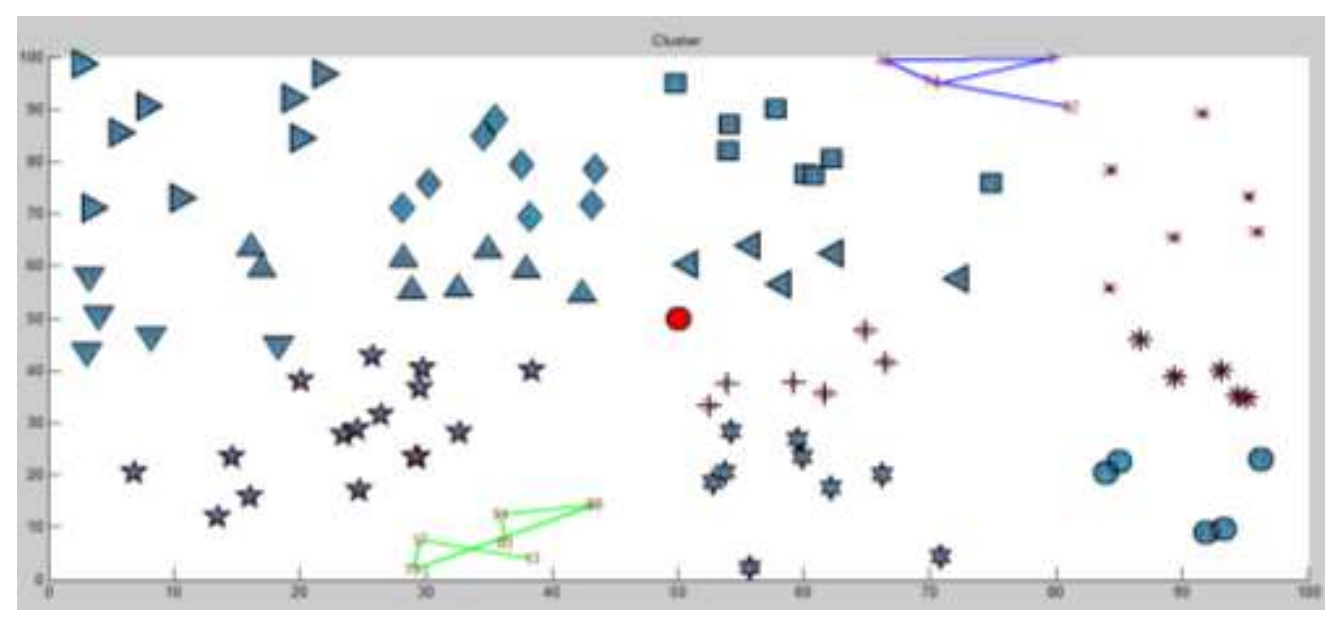

Fig 2. Fuzzy C-Means Clustering

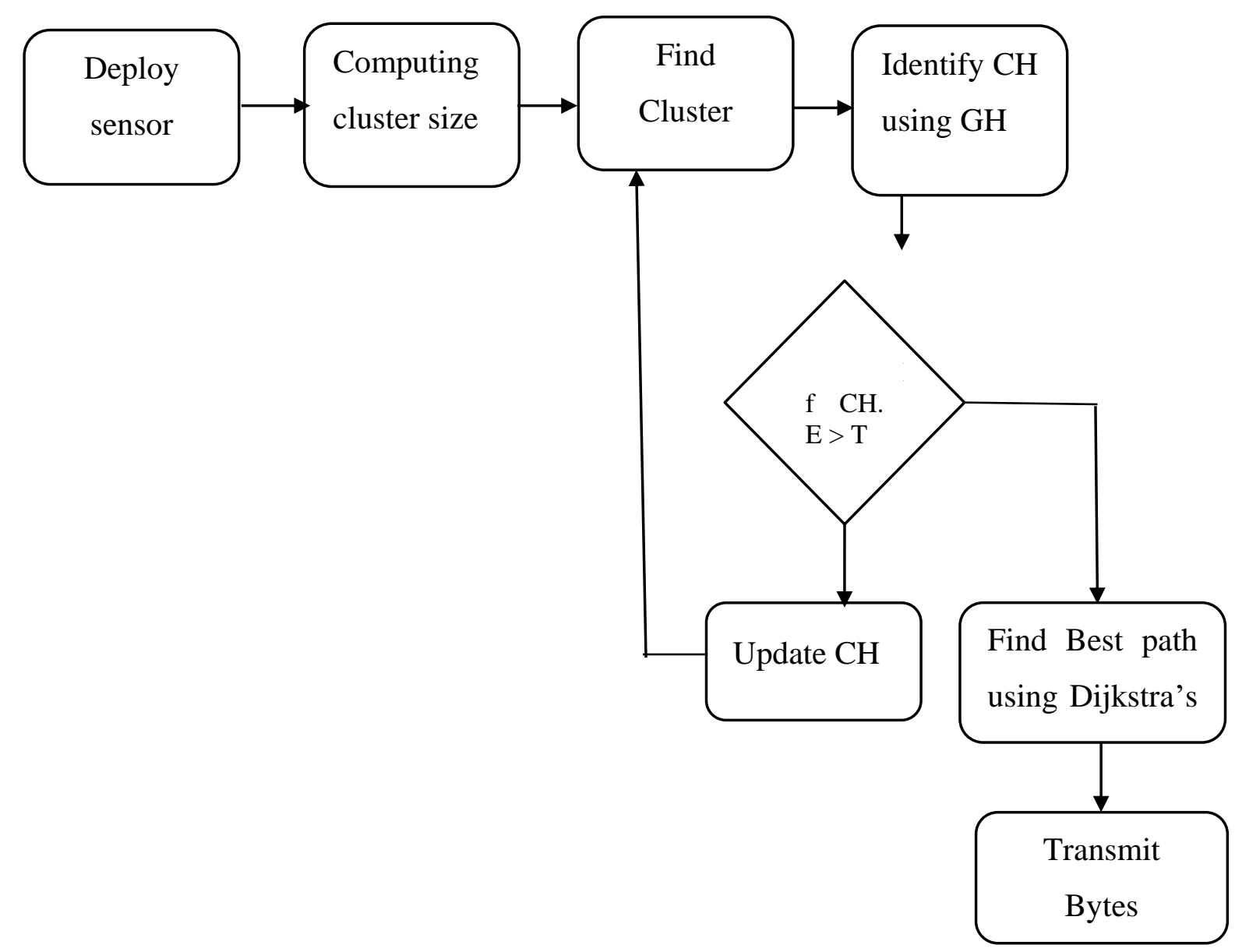

Fig 3. Proposed EFCRA Flow diagram 


\section{B. Identifying Cluster Head}

Cluster head $(\mathrm{CH})$ selection among in each cluster plays a vital role in IoT based WSN network. Several Parameters like residual energy, distance, mobility and density of nodes are considered for cluster head selection. IoT networks are more reactive and dynamic in nature. Therefore cluster head is selected as a node with more capability and higher energy compared to other node among the cluster. Greedy heuristic is an algorithmic technique that solves an optimization problem by finding locally optimal solutions. This approach is used to fix a cluster head where the basic cluster centres are formed by fuzzy $\mathrm{C}$ mean clustering. The distance between the cluster centres to all the other nodes that belongs to same cluster is obtained. The node which has minimum distance to all the other nodes in the same cluster is considered to be the $\mathrm{CH}$ node. Once the cluster head is fixed distance from the sensor node to the $\mathrm{CH}$ node and then to the base station is computed using the Dijkstra's algorithm.

To find the best route, distance from source to destination, and energy are considered. The weighted averaging is performed to get the best solution for the system. For the best path, distance should be minimum and corresponding cluster head should have high energy. The weight for distance and energy is equally assigned to get the best solution.

The energy of the cluster head is obtained and it should be greater than threshold value. The energy of $\mathrm{CH}$ node gets depleted when the number of rounds of transmission of data increases. When the energy of the cluster head is lesser than the threshold value, the next nearest node to this cluster head is considered to be the cluster head. The energy consumed during the transmission and reception of data between the nodes is given as

$$
E_{c(u)}=E T_{x(u)}+E R_{x(u)}
$$

$E T_{x}$ and $E R_{x}$ represents the energy that are consumed during transmission and reception of data. The transmission energy $E T_{x}$ and the reception energy $E R_{x}$ are defined as follows:

$$
\begin{aligned}
& E T_{x(k, d)}=E_{\text {elec*k}}+E_{\text {amp*k*d2 }} \\
& E R_{x(k, d)}=E_{\text {elec } * k}
\end{aligned}
$$

$E_{\text {elec }}$ means the electronic energy, $k$ denotes the Message length in bits, $E_{\text {amp }}$ represents the Transmission amplification coefficient, and $d$ signifies the distance between 
the sender node and the receiver node. The fig. 4 shows the Enhanced fuzzy based clustering and Routing Algorithm (EFCRA).

\section{EFCRA Algorithm}

\begin{tabular}{|c|c|}
\hline 1 & Input : Sensor vector $x_{i} i=1$ to $N$ \\
\hline 2 & Output: Optimized Cluster Head Ci \\
\hline 3 & For $\mathbf{i}=1$ to $\mathrm{N}$ do \\
\hline 4 & Find the cluster centroid for each sensor \\
\hline 5 & Calculate optimized centroid using \\
\hline 6 & $c_{j}=\left(\sum_{i=1}^{n}\left(\mu_{i j}\right)^{m} x_{i}\right) /\left(\sum_{i=1}^{n}\left(\mu_{i j}\right)^{m}\right)$, where $j=1,2, . . c$ \\
\hline 8 & Identifying the cluster head using Greedy Heuristic \\
\hline & Techniques \\
\hline 10 & Check current energy level of each sensor which is \\
\hline 11 & nominated for centroid by using the condition \\
\hline 12 & IF $(C H . e>T)$ then \\
\hline 13 & Select candidate $\mathrm{CH}$ \\
\hline 14 & ELSE \\
\hline 15 & Search Next CH candidate \\
\hline 16 & Calculate the distance between destination and cluster head \\
\hline 17 & Find the best solution using weighted average \\
\hline 18 & Select best path \\
\hline & Store best cluster head index $C_{i}$ \\
\hline 19 & End for \\
\hline 20 & Return $\mathbf{C}_{\mathbf{i}}$ \\
\hline
\end{tabular}

Fig.4 EFCRA Algorithm

\section{Results and Discussions}

The simulation of the proposed work is performed using MATLAB. Also performance analysis is compared with the multi-hop clustering technique [18]. The monitoring zone considered for this work is 25 X 25 square meter. Uniform random distribution of 25 to 500 number of sensors can be deployed in the monitoring zone. The simulation parameters are listed in Table 1. 
Table 1. Simulation Parameters

\begin{tabular}{|l|c|}
\hline \multicolumn{1}{|c|}{ Parameter } & Value \\
\hline Software & MATLAB \\
\hline Area & $25 \mathrm{mx} 25 \mathrm{~m}$ \\
\hline Number of Sensors & $25-500$ \\
\hline Distribution of nodes & Uniform Random \\
\hline Initial energy of each node $\mathrm{E}_{\text {init }}$ & $1 \mathrm{Joule}$ \\
\hline Electronic energy $\mathrm{E}_{\text {elec }}$ & $50 \mathrm{nj} / \mathrm{bit}$ \\
\hline Transmission amplification coefficient $\mathrm{E}_{\mathrm{amp}}$ & $100 \mathrm{pj} / \mathrm{bit} / \mathrm{n}^{2}$ \\
\hline Packet size & $1000 \mathrm{bits}$ \\
\hline
\end{tabular}

\section{A. Performance Metrics}

The following metrics are used for evaluating the performance of proposed algorithm.

\section{Network Lifetime}

It measures the operational time of the network from beginning untlil the death of the last node.

\section{Number of Alive Nodes per round}

It computes the number of nodes that have energy to transmit the data in each round.

\section{Number of Dead Nodes per round}

It measures the number of nodes without energy to transmit the data in each round

\section{Number of cluster heads per round}

It calculates the number of $\mathrm{CH}$ nodes formed in every transmission of message.

\section{Energy consumption node}

It measures the energy consumed by the network at each round of transmission.

The proposed algorithm shows better clustering and routing methods for communication among large scale IoT network. In EFCRA, coverage of IoT network is increased due to the fuzzy $\mathrm{C}$ mean clustering. Fig. 5 displays the result of number of alive nodes with number of rounds of data transmission. The proposed algorithm indicates that number of alive nodes is maximum when compared to the existing method. 


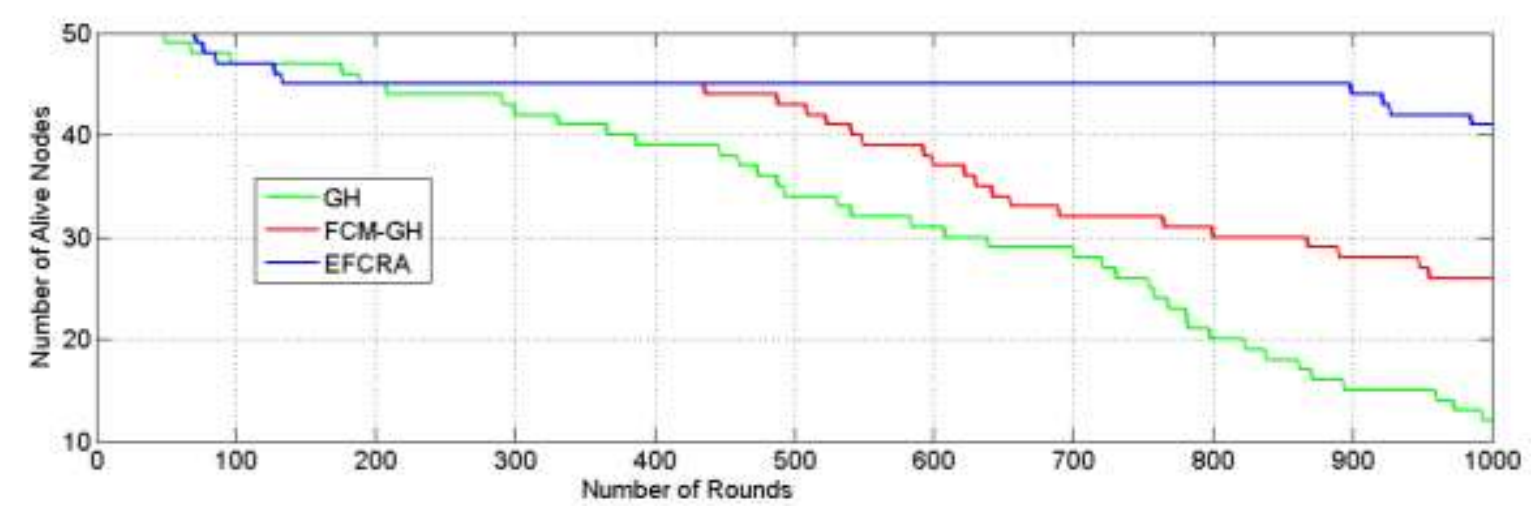

Fig. 5 Number of Rounds verses Number of Alive nodes

Fig. 6 shows that number of dead nodes is minimum in the EFCRA algorithm when number of rounds of transmission of messages increases.

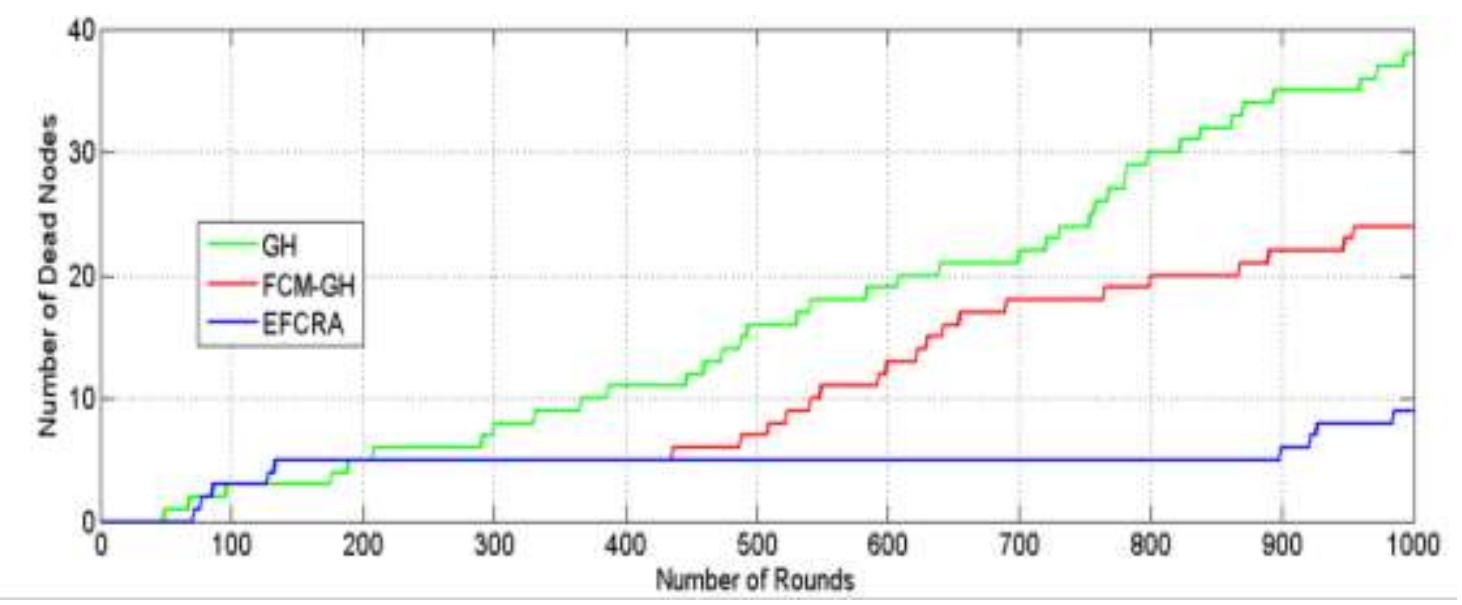

Fig.6 Number of Rounds verses Number of Dead nodes

Fig.7 depicts the number of alive nodes and dead nodes in both EFCRA algorithm and existing algorithm. The proposed algorithm provides the maximum energy efficiency because of more number of alive nodes and lesser number of dead nodes verses number of rounds. Also fig.7 displays that the proposed system attains a better performance than the existing system. 


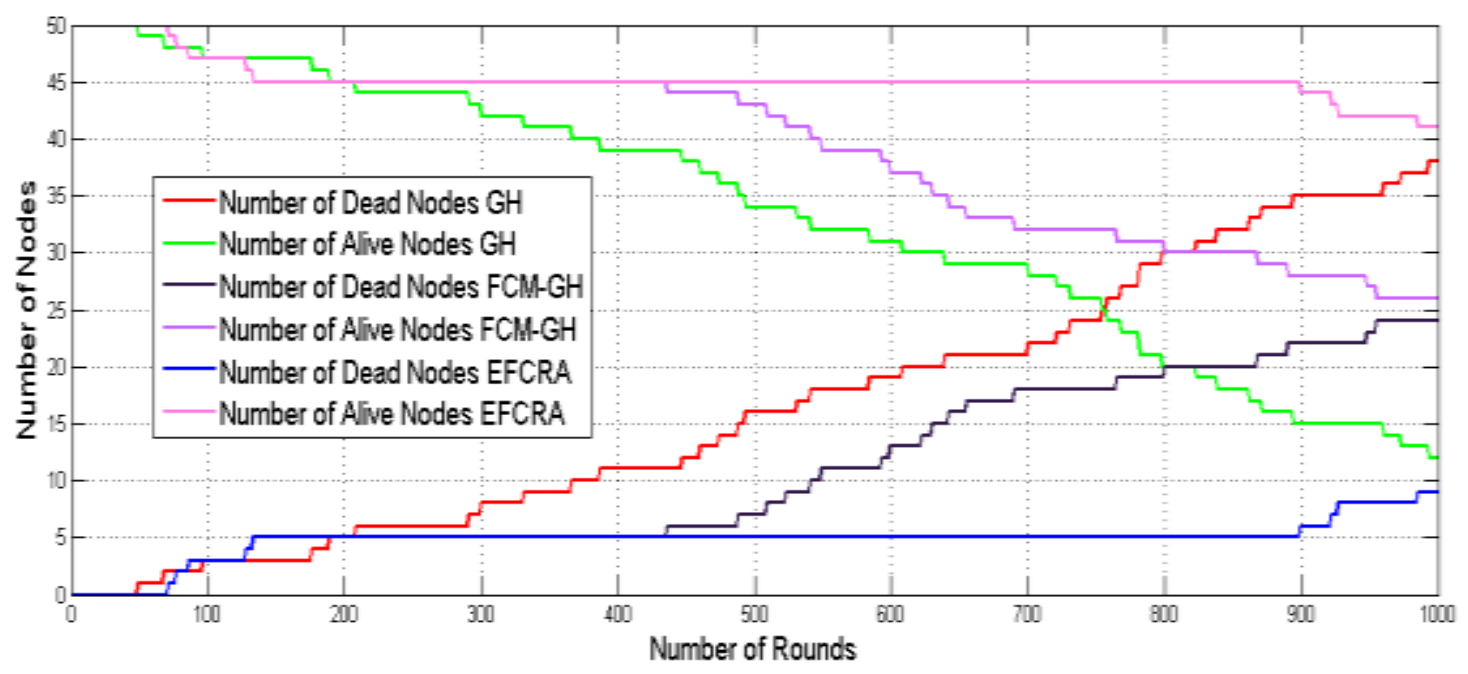

Fig 7. Number of rounds verses number of nodes

\section{B. Throughput}

Throughput measures how many packets arrived at their destinations over a communication channel successfully. People often are concerned about measuring the maximum data throughput in bits per second of a communications link or network access. Throughput can be found using the following equation:

$$
=I / T
$$

Fig. 8 shows the number of rounds verses throughput of the proposed algorithm and it attain the maximum throughput efficiency.

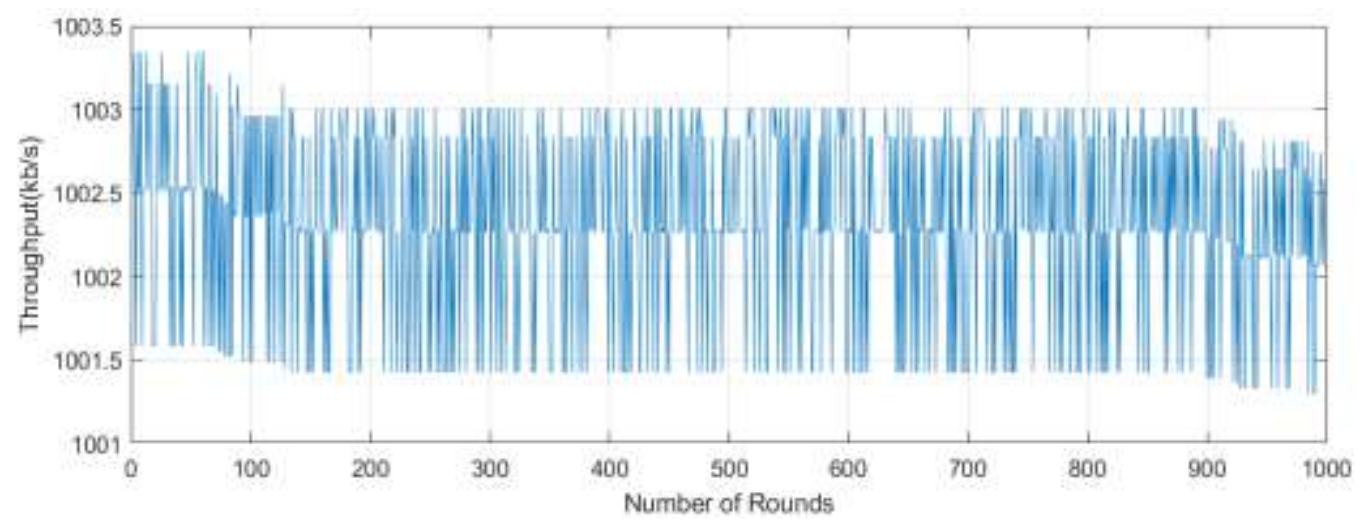

Fig.8 Throughput

\section{Comparative analysis}

The comparative analysis of alive nodes and dead nodes of EFCRA with hop count constraint-based clustering mechanism of nodes in IoT networks which minimize the number of required Internet connections [12] and FCM-GH is shown in table 2. 
Table 2. Comparison of Alive and Dead Nodes of GH, FCM-GH and EFCRA Algorithms

\begin{tabular}{|c|c|c|c|c|c|c|}
\hline \multirow{2}{*}{$\begin{array}{c}\text { Number of } \\
\text { Rounds }\end{array}$} & $\begin{array}{c}\text { No. of } \\
\text { Alive } \\
\text { nodes }\end{array}$ & $\begin{array}{c}\text { No. of } \\
\text { Dead } \\
\text { Nodes }\end{array}$ & $\begin{array}{c}\text { No. of } \\
\text { Alive nodes }\end{array}$ & $\begin{array}{c}\text { No. of } \\
\text { Dead } \\
\text { Nodes }\end{array}$ & $\begin{array}{c}\text { No. of Alive } \\
\text { nodes }\end{array}$ & $\begin{array}{c}\text { No. of } \\
\text { Dead } \\
\text { Nodes }\end{array}$ \\
\hline 1 & 50 & 0 & 50 & 0 & 50 & 0 \\
\hline 100 & 47 & 3 & 47 & 3 & 47 & 3 \\
\hline 200 & 45 & 5 & 45 & 5 & 45 & 5 \\
\hline 300 & 42 & 8 & 45 & 5 & 45 & 5 \\
\hline 400 & 39 & 11 & 45 & 5 & 45 & 5 \\
\hline 500 & 34 & 16 & 44 & 6 & 45 & 5 \\
\hline 600 & 31 & 19 & 36 & 14 & 45 & 5 \\
\hline 700 & 29 & 21 & 32 & 16 & 45 & 5 \\
\hline 800 & 20 & 30 & 30 & 20 & 45 & 5 \\
\hline 900 & 15 & 35 & 28 & 22 & 45 & 5 \\
\hline 1000 & 12 & 38 & 26 & 24 & 42 & 8 \\
\hline
\end{tabular}

Here in table 2, 1 to 1000 rounds of data transmission are considered. The proposed method contains more number of alive nodes and less number of dead nodes in each round when compared to the existing method. The proposed mechanism achieved $84 \%$ of alive nodes thus providing better performance of the network.

\section{CONCLUSION}

The proposed algorithm enhances the quality of service by improving the coverage and throughput of IoT based WSN network. The algorithm uses Fuzzy c means based clustering of sensor nodes to improve the coverage in the network. It also checks the energy of the sensor nodes and cluster head node during the transmission of messages. The cluster head node is selected based on node with minimum distance and maximum energy. The algorithm also provides maximum energy efficiency that increases the network lifetime. The increased network lifetime leads to enhanced QoS and successful arrival of packets to the destination results in maximum throughput in the network. 


\section{REFERENCES}

[1] J Shen, A Wang, C Wang, P C Hung, C F Lai, "An efficient centroid-based routing protocol for energy management in WSN-assisted IoT," IEEE Access. 2017; Vol. 5. pp. 18469-18479.

[2] JV Sobral, JJ Rodrigues, RA Rabelo ,JC Lima Filho, N Sousa, H S Araujo, R. Holanda Filho. "A framework for enhancing the performance of Internet of Things applications based on RFID and WSNs", Journal of Network and Computer Applications. 2018 Apr 1; 107:56-68.

[3] S Ezdiani, IS Acharya, S Sivakumar, A Al-Anbuky, "Wireless sensor network softwarization: towards WSN adaptive QoS," IEEE Internet of Things Journal, 2017 Aug 29; 4(5):1517-27.

[4] H Mostafaei, "Energy-efficient algorithm for reliable routing of wireless sensor networks," IEEE Transactions on Industrial Electronics. 2018 Sep 13; 66(7):556775.

[5] M Manisha Singh and G Baranwal, "Quality of Service (QoS) in Internet of Things", 3rd International Conference on Internet of Things: Smart Innovation and Usages (IoT-SIU), IEEE 2018.

[6] Rania Khadim, Abdelhakim Maaden, Ansam Ennaciri, Mohammed Erritali, “An Energy-Efficient Clustering Algorithm for WSN Based on Cluster Head Selection Optimization to prolong Network Lifetime", International Journal of Future Computer and Communication, vol 7,No.3,September2018

[7] S Su and S Zhao, "An optimal clustering mechanism based on Fuzzy-C means for wireless sensor networks", Sustainable Computing: Informatics and Systems,2018 Jun 1;18:127-34.

[8] K Thangaramya, K Kulothungan, R Logambigai, M. Selvi,S Ganapathy,A Kannan , "Energy aware cluster and neuro-fuzzy based routing algorithm for wireless sensor networks in IoT”, Computer Networks, 2019 Mar 14;151:211-23.

[9] S.K.S.L Preeth, R Dhanalakshmi, R Kumar , P. M Shakeel, “An adaptive fuzzy rule based energy efficient clustering and immune-inspired routing protocol for WSN-assisted IoT system", Journal of Ambient Intelligence and Humanized Computing. 2018:1-3.

[10] JS Raj, "QoS optimization of energy efficient routing in IoT wireless sensor networks. Journal of ISMAC", 2019; 1(01):12-23. 
[11] L. Xu, R. Collier and G. M. P. O’Hare, "A Survey of Clustering Techniques in WSNs and Consideration of the Challenges of Applying Such to 5G IoT Scenarios," in IEEE Internet of Things Journal, vol. 4, no. 5, pp. 1229-1249, Oct. 2017.

[12] Sung, Yoonyoung, Sookyoung Lee, Meejeong Lee, "A multi-hop clustering mechanism for scalable IoT networks”, Sensors 18.4 (2018): 961.

[13] Amol V. Dhumane, Rajesh S. Prasad, Jayashree R. Prasad, "An optimal routing algorithm for Internet of Things Enabling Technologies", International Journal of Rough set and Data Analysis, 2017 4(3).

[14] Xu, Lina, Gregory O'Hare, and Rem Collier, "A smart and balanced energyefficient multihop clustering algorithm (smart-beem) for mimo IoT systems in future network", Sensors 17.7 (2017): 1574.

[15] Anagha Rajput and Vinoth Babu Kumaravelu, "Scalable and sustainable wireless sensor networks for agricultural application of Internet of things using fuzzy cmean algorithm," Sustainable computing: Informatics and system”, Elsevier 2019, vol.22,

[16] K. Jaiswal and V. Anand, "An Optimal QoS-aware multipath routing protocol for IoT based Wireless Sensor Networks," 2019 3rd International conference on Electronics, Communication and Aerospace Technology (ICECA), Coimbatore, India, 2019, pp. 857-860, doi: 10.1109/ICECA.2019.8822173.

[17] Dr. D. I. George Amalarethinam and P. Mercy, "CHS_QoS: Cluster Head Selection using QoS Properties for Heterogeneous IoT based WSN", Malaya Journal of Matematik-An International journal of Mathematical Sciences with Computer Applications, Vol. S, No. 1, 298-303, 2021

[18] M. E. Khanouche, Y. Amirat, A. Chibani, M. Kerkar and A. Yachir, "EnergyCentered and QoS-Aware Services Selection for Internet of Things," IEEE Transactions on Automation Science and Engineering, vol. 13, no. 3, pp. 12561269, July 2016, doi: 10.1109/TASE.2016.2539240.

[19] https://sites.google.com/site/dataclusteringalgorithms/fuzzy-c-means-clusteringalgorithm 